\title{
Recognition of Vehicle Registration Mark on Moving Vehicles in an Outdoor Environment
}

\author{
N. H. C. Yung*, K. H. Au \& A. H. S. Lai \\ Laboratory for Intelligent Transportation Systems Research \\ The University of Hong Kong \\ Pokfulam Road, Hong Kong SAR \\ *nyung@eee.hku.hk
}

\begin{abstract}
In this paper, we propose a new method for segmenting vehicle registration plate and recognizing the registration mark on a moving vehicle in an outdoor environment. The algorithm first segments the plate from the vehicle and other complex objects in view, based on the plate color and its dimension. The segmented plate is then corrected in orientation and size, before being matched with a sub-set of templates in the database. The matching adopts a high tolerant scheme allowing characters to have certain degree of shifting, rotation and mis-match. This proves to be an important criterion for successful recognition. Preliminary tests show that this method offers high success rate and high confident level.
\end{abstract}

\section{Introduction}

Vehicle identification is a vital link in a number of Intelligent Transportation Systems (ITS) applications, including electronic road pricing, ramp metering, red-light runner detection, car parking and many others where exact vehicle identity is required. In some cases, this is feasible when the identification environment can be controlled. For example, in the case of car park or toll gate, the camera may be positioned such that its field of view (FOV) has the best coverage of the vehicle registration plate (VRP) of a stationary vehicle, where VRP here is defined as the plate with the vehicle registration mark (VRM) displayed on it. Lighting may also be adjusted in a limited sense to improve image contrast.

In other cases, such as electronic road pricing and redlight running, the application environment is poorly defined. Vehicles are most likely in motion on the road, where the FOV would probably be covering the whole vehicle rather than just the VRP. Apart from not being able to control much of the lighting during image capturing, the camera shutter speed has to be fast enough to eliminate motion blurring, which also reduces the illumination dynamic range of the image acquired. Besides, the segmentation has to deal with extracting the VRP from a complex scene and the recognition has to handle problems such as poor resolution, rotation and shifting, among others.
Essentially, the research in segmenting the VRP is not well established as the difficulty of the problem depends on whether the front or back VRP is captured, and whether the vehicle is freely in motion or restricted in position. In the former case, the front VRP is better defined while the back VRP does not necessarily has a fixed and defined location. In the latter, recognition of VRM on a stationary, positionrestricted vehicle would be easier to achieve [1-2]. In theory, traditional segmentation techniques are applicable here.

On the issue of VRM recognition, a number of approaches has been published [3-4], of which most of them deal with an indoor, well-controlled scenario. When outdoor cases are concerned, Yoshikawa et al [5] presented a recognition system used at toll-gates. They used a wide dynamic range camera positioned to view the front license plate of vehicles. At the toll-gate, vehicles are restricted in position and stationary. Therefore, their camera could have a close-up view of the license plate and thus giving high success rate.

In this paper, we propose a new method for dealing with the segmentation of VRP and recognition of VRM on a moving vehicle in an outdoor environment, with the camera viewing from behind. The algorithm first segments the VRP from the vehicle and other complex objects in view, based on the plate color and its dimension. The segmented VRP is then corrected in orientation and size, before being matched with a sub-set of templates in the database. The matching itself adopts a high tolerant scheme allowing characters to have certain degree of shifting and rotation. This proves to be an important criterion for successful recognition. Preliminary tests show that this method offers high success rate and high confident level.

This paper is organized as follows: Section 2 outlines the problem domain and the algorithm in general. Section 3 describes the VRP segmentation approach. Section 4 discusses the image processing techniques employed to enhance the VRP quality before matching. Section 5 outlines how the templates are constructed. Section 6 details the template matching process and presents some of the results. Section 7 concludes the paper. 


\section{Algorithm overview}

First, let us outline the problem of segmenting the VRP and recognizing the VRM on a moving vehicle in an outdoor environment, with the camera viewing from behind. Let us also use the VRP/VRM in Hong Kong as an illustration because different countries have different formats altogether.

In the case of Hong Kong, the back VRP is yellow in color with the VRM in black. The VRM is a combination of upper-case alphabets and numerals. A valid VRM can have between zero to two alphabets and a maximum of four numerals. They could be arranged on one line or two lines. There appear to have no standard fonts although the variation is not extensive, and B.S.145a stipulates the maximum and minimum size. We shall term both the alphabets and numerals as 'character' in the subsequent discussion for convenience.

Regarding the VRP mounting, there is no restriction as to where it should be at the back of the vehicle. In general, its between the two brake lights. However, there are numerous cases where the VRP is mounted at the most unusual places, such as near the top or under the bumper.

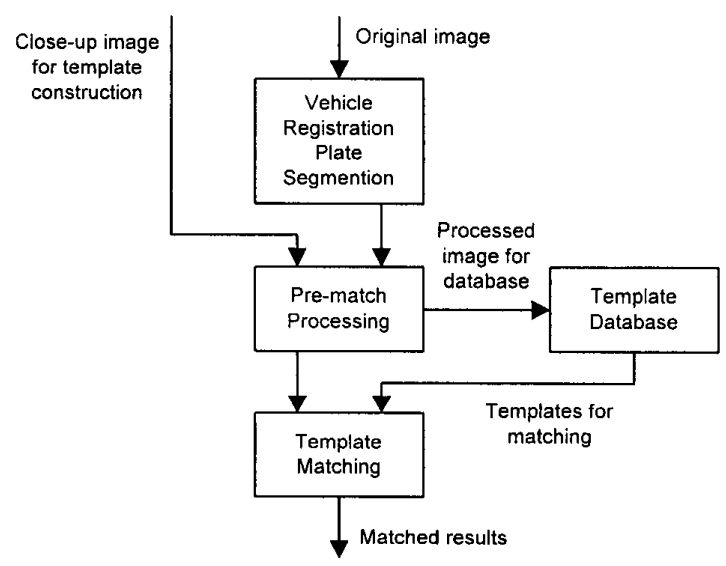

Figure 1. Proposed recognition algorithm.

With reference to Figure 1, the proposed algorithm consists of four major modules: VRP segmentation, prematch processing, template database and template matching.

In principle, segmentation of the VRP is performed on the whole digital image converted from a high-resolution photographic image. It consists of four steps: sub-sampling, search area reduction and yellow region detection. This approach combines data reduction and feature extraction to give a reliable segmentation of the VRP.

The pre-match processing (PMP) consists of a series of image processing steps for preparing the segmented VRP for recognition. This includes orientation correction to correct slanting due to perspective view, line segmentation to determine one or two line VRP, thresholding to simplify subsequent processing, morphological closing to remove holes and ruggedness, character segmentation to isolate characters and scaling to resize the character to those of the templates.
The template database contains the templates of 21 numerals and 28 characters for template matching in the latter stage. These templates are constructed similarly as in the recognition of them.

Template matching performs the recognition by first using the number of holes in the character to pick a subclass. Then all the templates in a sub-class is matched with the input character. A confidence figure is calculated in each case, which is bounded between 100 (perfect match) and 0 . Recognition is thus achieved by choosing the one with the highest probability.

\section{VRP segmentation}

From Figure 2, the purpose of sub-sampling is to reduce the image size by 16 times for the subsequent steps. This is because the photographic image has very high spatial resolution designed for visual recognition of the VRM. From the sub-sampled image, search area reduction is performed to further reduce the amount of data involved. Specifically, this step searches for the top and bottom of the vehicle, or other features that can achieve similar effect. For example, in the case of red-light runner detection, stop line detection and red light detection are used for this purpose [6]. If there is no significant features available in the image, this step may be skipped.

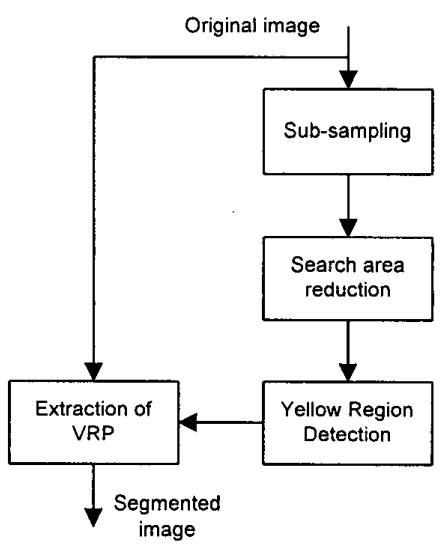

Figure 2. VRP segmentation.

The detection of the yellow VRP is achieved by first finding all the yellow regions in the image, then retaining the yellow regions with width-to-height ratio between 1.4 and 2.5 or between 4.0 and 5.5. These two ranges denote the two-line VRP and one-line VRP respectively. The equations for identifying different color regions are given below, where Eqt ( 3 ) is used to identify the yellow regions:

$$
\begin{aligned}
& R_{W, k}=\left\{g(x, y) \in I_{l}: g_{S}(x, y)<t_{s} \text { and } g_{V}(x, y)>t_{v}\right\} \\
& R_{R, k}=\left\{g(x, y) \in I_{1}:\left|g_{H}(x, y)\right|<\varepsilon \text { and } g_{V}(x, y)>t_{v}\right\} \\
& R_{Y, k}=\left\{g(x, y) \in I_{l}:\left|g_{H}(x, y)-\frac{\pi}{3}\right|<\varepsilon \text { and } g_{V}(x, y)>t_{v}\right\} \\
& R_{G, k}=\left\{g(x, y) \in I_{l}:\left|g_{H}(x, y)-\frac{2 \pi}{3}\right|<\varepsilon \text { and } g_{V}(x, y)>t_{v}\right\}(4)
\end{aligned}
$$


where $t_{s}, t_{v}$ and $\varepsilon$ are threshold values; $R_{\psi, k}$ are connected regions that $R_{\psi, k} \cap R_{\psi, j}=\phi$, for all $k$ and $j, k \neq j$ and $\psi=W$, $R, Y, G$. The coordinates of the detected yellow regions in the sub-sampled image are then used to segment the VRP from the original image. If there are more than one yellow regions that satisfy the above criteria, all those regions will be considered for pre-match processing and VRM recognition. Figure 3 depicts part of the original image and the segmented VRP.

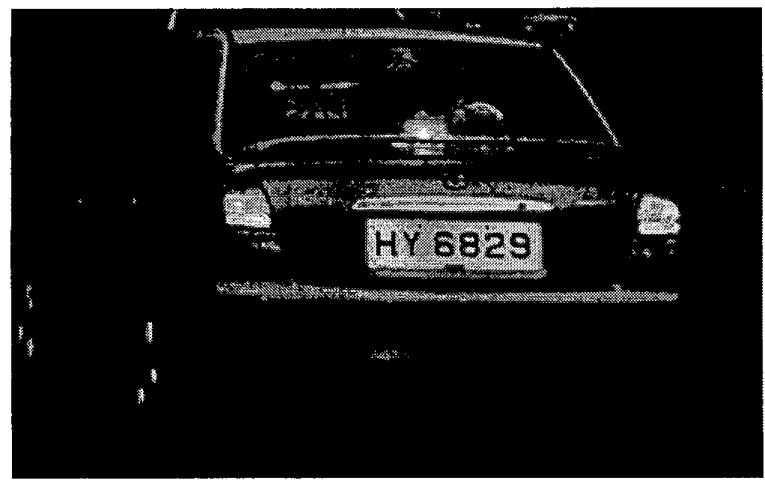

(a) Part of the original image

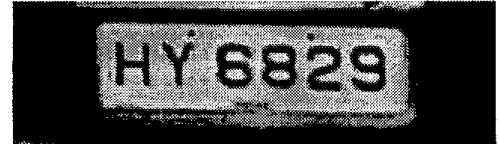

(b) Segmented image

Figure 3. Segmented VRP.

\section{Pre-match processing}

As can be seen in Figure 3, the segmented VRP is distorted because of the perspective view. Instead of performing a perspective correction, we correct the orientation by determining the top, bottom, left and right boundary lines of the yellow region. Based on these lines, the image is transformed accordingly. These are shown in Figure 4. Details of the PMP are depicted in Figure 5.

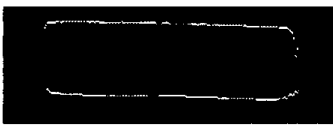

(a) top and bottom lines

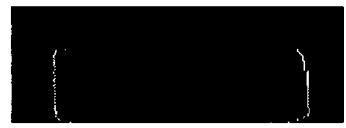

(b) Left and right lines

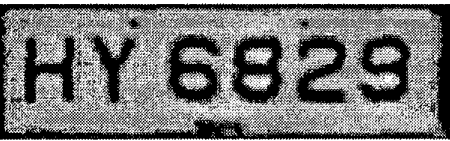

(c) Corrected VRP

Figure 4. Orientation correction.

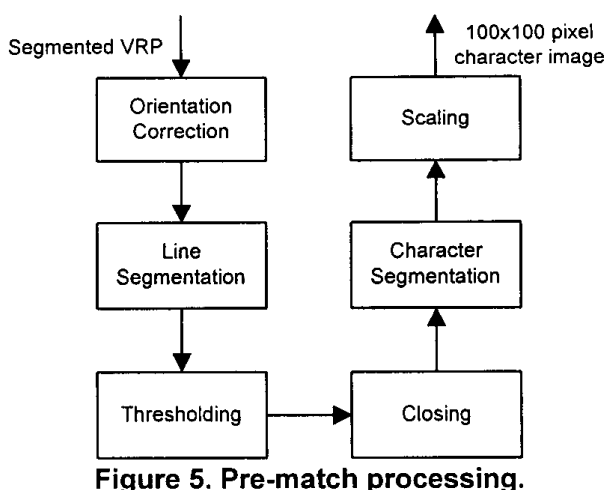

If the VRP has two lines, we then find three values: the lowest point of the second line, the top point of the first line and the height of the second line. These values enable us to divide the two lines.

After this, the line image is thresholded into binary image using the following equation:

$$
T=\frac{1}{2}\left(f_{b}+f_{d}\right)
$$

where $f_{b}$ is the average intensity of the brightest line and $f_{d}$ is the average intensity of the darkest line. The next step is to use morphological closing to remove the small holes and smooth the ruggedness of the characters. The results of both cases are depicted in Figure 6(a) and (b).

To segment individual character out of the image depicted in Figure 6(b), $x$ - and $y$-projected histograms are obtained to determine the number of characters on the line and where to separate them. If the character width is too narrow or the height of the character is too short, it will be declared a non-character. Based on this information, each character is segmented. The final scaling is simply done by re-sizing the character to $100 \times 100$ pixels with the height of the character fitting the vertical 100 pixels, while retaining the width-to-height ratio of the character. A typical case is depicted in Figure 6(c)

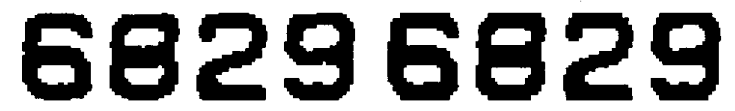

(a) thresholded

(b) after closing

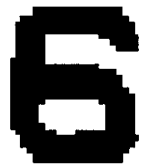

(c) segmented and scaled character Figure 6. Processed character.

\section{Construction of templates}

The set of templates for recognition consists of 21 numerals and 28 upper-case characters. The reason for having more than 26 characters is because there are quite a 
few different fonts used for VRM in Hong Kong. The extra templates are constructed to ensure a high recognition rate.

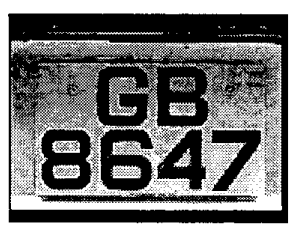

Figure 7. Close-up VRP for template construction.

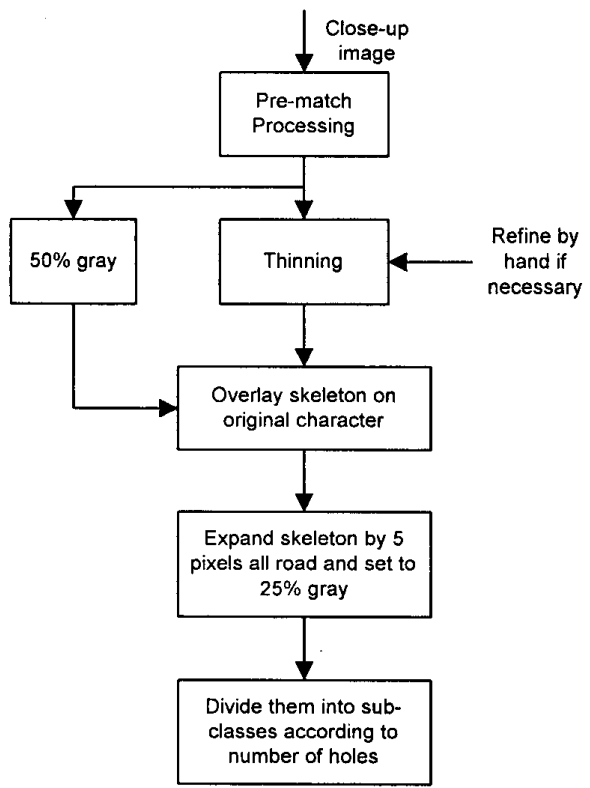

Figure 8. Template construction.

To construct the templates, first, close-up digital images of different VRP are acquired. A typical one is shown in Figure 7. As in Figure 8, the VRP is processed by the PMP module as described in Section 4, and individual characters are extracted. It is then thinned into a skeleton, which may be edited by hand if necessary. If white is 1 and black is 0 , the pixels of the skeleton is assigned to black (0), while the original image is assigned to $50 \%$ gray $(0.5)$. The skeleton is then overlaid on the gray image. The boundary of the $50 \%$ gray character is further expanded by 5 pixels with assigned gray values of 0.75 . This forms the template of one character. In summary, a template has four levels: black (skeleton), $50 \%$ gray (original character), $25 \%$ gray (expanded character outline) and white. The rest of the template database is compiled in exactly the same way. Figure 9 depicts some of the templates.

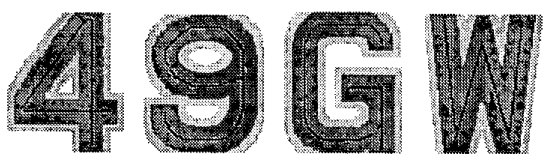

Figure 9. Templates of '4', '9', 'G' \& 'W'.

\section{VRM recognition}

The first step in template matching is to fill the black or white holes in the scaled character image. This is done by expanding the boundary of the $100 \times 100$ pixels image by 1 pixel on each boundary. This causes the white region external to the character to merge and form one large region. For black regions less than 100 pixels, or white region less than 70 pixels, their corresponding values are inverted. The reason for doing hole filling is that such small holes are common in images acquired in this way. On the other hand, legitimate holes are usually bigger than these values. After this, the number of holes internal to the character is counted. This is used to invoke the corresponding sub-class of characters in the matching stage. Details of this process are depicted in Figure 10.

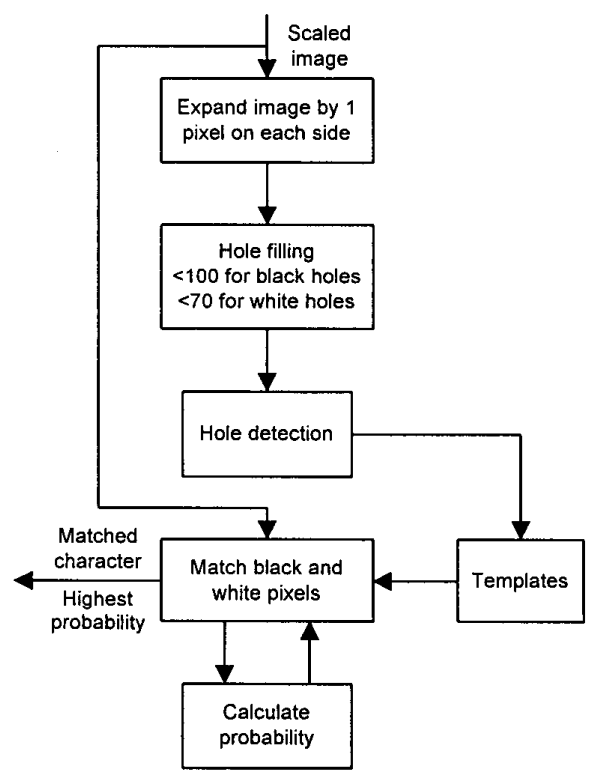

Figure 10. Template matching.

In matching the templates, we compare the input character with the templates in the sub-class pixel-by-pixel. The pixel comparison is only performed on locations at the black pixels (skeleton) and white pixels on the template. This method gives a degree of tolerance to the input character being shifted, slightly rotated or not exactly the same as the template. A probability value is then calculated using the following equation:

$$
P=\frac{B_{m}}{B_{l}} \times \frac{W_{m}}{W_{l}}
$$

where $B_{m}=$ number of black points in the template that matches with the character; $W_{m}=$ number of white points in the template that matches with the character; $B_{t}=$ total number of black points in the template; $W_{1}=$ total number of white points in the template. Figure 11 depicts three cases. 


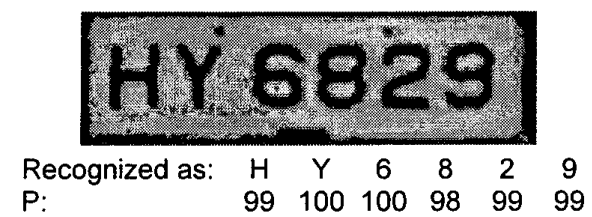

(a) Successful result

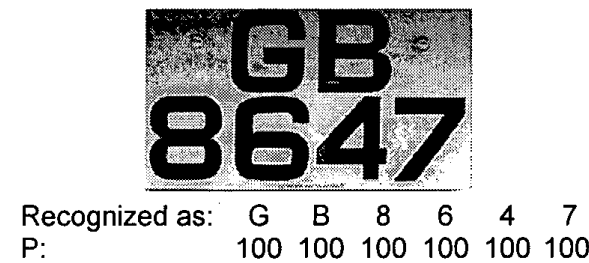

(b) Successful result

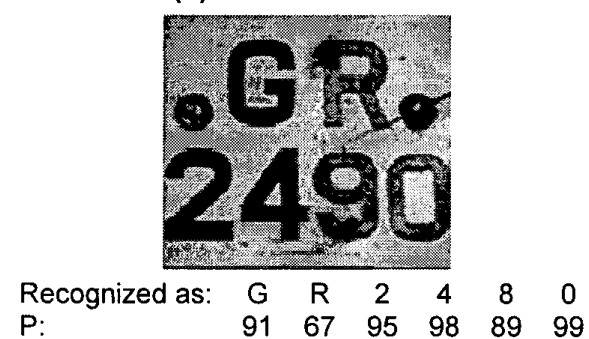

(c) Failed result

Figure 11. Matching results.

From Figure 11, it can be seen that the algorithm handles the desired input VRP very well (Figure 11(a)). Although the characters look blurred and slightly rotated, the recognition was all correct and the highest $\mathrm{P}$ is $100 \%$ and lowest $\mathrm{P}$ is $91 \%$. For an almost ideal input as shown in Figure 11(b), the recognition result was perfect. However, when the input is strongly affected by the quality of the VRP and the lighting, the recognized results could deviate from the normal cases. The VRP shown in Figure 11(c) was deliberately chosen which has both ' $R$ ' and ' 9 ' affected by dirt and the VRP is brighter on the right than on the left. Because of this, ' 9 ' was mistakenly recognized as ' 8 ' and although ' $R$ ' was correctly recognized, its probability is only $67 \%$, which is much lower than the other successful cases presented here. Detailed and extensive performance analysis is underway to determine the success rate of this recognition algorithm. So far with the various VRP tested, we believe the success rate would be substantially higher than $95 \%$.

\section{Conclusion}

From the above results, it can be concluded that the new recognition method is able to recognize vehicle registration mark of a moving vehicle in an outdoor environment. The vehicle registration plate segmentation is effective in extracting the VRM out of a rather complex scene. Moreover, the pre-match processing is able to enhance the image quality for the best possible matching eventually. Furthermore, the template matching offers high tolerance to character shift, rotation and imperfection. Finally, we believe the algorithm is able to give a very high success rate.

\section{References}

[1] J. A. G. Nijhuis et al., "Car license plate recognition with neural networks and fuzzy logic", Proceedings of the IEEE International Conference on Neural Network, vol. 5, pp. 2232-2236, 1995.

[2] Joon Y. Min and Jong U. Choi, "Development of license plate extraction algorithm using prior knowledge", Proceedings of the $5^{\text {th }}$ World Congress on ITS, 1998.

[3] S. K. Kim et al., "A recognition of vehicle license plate using a genetic algorithm based segmentation", Proceedings of the International Conference on Image Processing'96, vol. II, pp. 661-664, 1996.

[4] Y. H. Hwang et al., "A study on automatic identification of vehicle license plate", Proceedings of the Joint Conference on Signal Processing, vol. 7, no. 1, pp. 433-437, 1994.

[5] Yoshikawa et al, "Vision systems for ITS using wide dynamic range camera - application in license plate recognition system, parking lot monitoring system", Proceedings of the $5^{\text {th }}$ World Congress on ITS, 1998.

[6] Lai A. H. S. \& N. H. C. Yung, "A video-based system methodology for detecting red light runners", Proceedings of the IAPR Workshop on Machine Vision Applications, ISBN 4-091122-98-3, pp.23-26, 1998. 